work-like and flocculent appearances so often observed in the froth which is formed when the tide breaks on the seashore may be explained in a similar manner. HAROLD WAGER.

West Park, Leeds, April r4.

\section{An Extension of the Spectrum in the Extreme Ultra-Violet.}

THE researches of Schumann led him to extend the spectrum to the neighbourhood of wave-length 1250. His limiting wave-length was determined by the absorption of the fluorite which formed a necessary part of his apparatus. In $1904 \mathrm{I}$ succeeded in pushing the limit to wave-length Iozo by the use of a concave diffraction grating.

Recently I have renewed the attack on the problem, with the result tilat I have succeeded in photographing the spectrum of hydrogen to wave-length 905 . The extension is due, not so much to any fundamental change in the nature of the apparatus as to an improvement in technique consequent on an experience of ten years.

It is a characteristic of the region investigated by Schumann between wave-lengths 1850 and I250 that, while hydrogen yieıds a rich secondary spectrum, with the possible exception of one line, no radiation has been discovered belonging to the primary spectrum. On the other hand, in the new region between the limit set by fluorite and wave-length 905 , a disruptive discharge in hydrogen produces a primary spectrum of great interest made up of perhaps a dozen lines. These lines are always accompanied in pure hydrogen by members of the secondary spectrum, but they may be obtained alone if helium containing a trace of hydrogen is employed.

Results obtained from vacuum tubes when a strong disruptive discharge is used, must always be interpreted with caution since the material torn from the tube itself sometimes furnishes impurities. In the present case, it will be some time before the effect of such impurities can be estimated. However, it may be stated with some degree of certainty that the diffuse series predicted in this region by $\mathrm{Ritz}$ has been discovered. The first member at 1216 is found to be greatly intensified by the disruptive discharge, and the next linc at 1026 appears also, though very faintly. This diffuse series bears a simple relation to Balmer's formula. Following the same kind of argument, a sharp series corresponding to the Pickering series might be expected. The new region appears to yield two lines belonging to such a relation at the positions demanded by calculation.

Theodore LymaN.

Harvard University, April 20.

\section{The Structure of Atoms and Molecules.}

Since in an elaborate criticism of Bohr's theory on the constitution of atoms and molecules, Prof. J. W. Nicholson, as in his letter to Narure (February 5, p 630), comes to the conclusion (Phil. Mag., xxvii., p. 560 , I914) that the valencies of lithium, beryllium, boron, etc., on Bohr's theorv are not in accord with experience, and if the electrons in the atoms are to be in one plane, we must either abandon Bohr's method of calculating valency-and (generally) Bohr's theory of the atoms more complex than hydrogen and helium-or give up van den Broek's hypothesis, that the charge of the nucleus of Rutherford's atom is equal to the atomic number (which hypothesis was accepted by Bohr as one of his fundamental assumptions), I may be allowed to add some remarks to my previous letter on this subject (Nature, March 5, 1914).

NO. 2323 , VOL. 93]
For these atoms at least this hypothesis is a mere expression of experimental facts. The hydrogen atom is known to lose never mote than one electron, and the helium atom never more tnan two, and, of course, never one to form an electrolytic ion, while lithium, beryllium, boron, and carbon can lose, or, in chemical combination, dispose of $\mathrm{I}, 2,3,4$ electrons respectively. Further, the number of electrons per atom has been proved to be nearly equal to half the atomic weight (Rutherford, Barlslo), and in the case of carbon to be six (Rutherford, Phil. Mag., vol. xxvi., p. 7i I, Igr3). Since the number of electrons per atom must be an integer, here, at least, no other solution seems to be possible than that the number of electrons per atom surrounding the nucleus, and hence the nuclear charge, is equal to the atomic number.

Further mentioning Moseley's previous experiments on high-frequency spectra (undertaken for the express purpose of testing the atomic number hypothesis), and criticising the theoretical deductions, derived by Moseley from these experiments, Nicholson concludes that they have shown no relation to Bohr's theory (loc. cit., p. 564). Now in another paper Moseley, from further experiments on high-frequency spectra, proves ( $P$ hil. Mag., vol. xxvii., p. 703, I9I4) that the frequency of any line in the X-ray spectra is approximately proportional to $\mathrm{A}(\mathrm{M}-b)^{2}$, where $\mathrm{A}$ and $b$ are constants for each series, and $M$, the atomic number (called by Moseley $\mathrm{N}$ ) of the element, is identified with the num. ber of positive units of electricity contained in the atomic nucleus, so that these experiments "give the strongest possible support" to this atomic number hypothesis (loc. cit., p. 712). The number of rareearth elements as given by Moseley is the only exception.

That $b$ is much larger for he " $L$ " lines than for" the " $\mathrm{K}$ " lines suggests, according to Moseley (in agreement with my own views, NATURE, December 25. 1913) that the "L" system is situated the further from the nucleus. If so, $b=$ the number of electrons nearest the nucleus, and not $=\sigma_{n}$, the term arising from the influence of the electrons in a ring on each other, and, for the " $\mathrm{K}$ ' lines, $n$, like $b$, must be unity, as calculated by Nicholson on Bohr's theory. For the "L" lines, according to Moseley, $b=7 \cdot 4$, but it can easily be seen from the tables that if $(M-b)$ be here augmented by 0.8 per cent., all values are integers $( \pm 0 \cdot 2)$, and $b=7$ and $n=1$ again, but perhaps the factor $5 / 36$ in Moseley's interpretation cannot be retained.

Hence, though this number 7 requires confirmation, principally, for the " $\mathrm{K}$ " line at least, Bohr's theory is here in agreement with Moseley's experiments, and with the atomic number hypothesis. Not only the frequencies, but also the minimum velocity of electrons requited to excite this radiation, and the absorption of it (in aluminium) have been proved (loc. cit.) to depend on the atomic number very nearly, and Nicholson's conclusion that the atomic numbers are not correct does not hold, for $(\mathrm{M}-b)$, not $\mathrm{M}$, is one unit less for the $\mathrm{K}$ radiation than the corresponding atomic number. But, from analogy, Bohr's lithium atom, as well as Nicholson's ring of three electrons, must be given up, for of three, one electron $(b)$ must be very near the nucleus, one $(n)$ near but outside this first one, and one as electron of valency must be peripheric.

Further, the velocity of electrons, required to excite this radiation, according to Widdington equal to IO $^{8} \times$ atomic weight $\mathrm{cm}$. $/ \mathrm{sec}$, is more accurately equal to $2.24 \times 10^{8}(\mathrm{M}-\mathrm{I}) \mathrm{cm}$. / scc., than for $\mathrm{Cr}, \mathrm{Fe}, \mathrm{Ni}, \mathrm{Cu}$, $\mathrm{Zn}$, and $\mathrm{Se}$; the last formula gives for the constant reduced to unity $0.99, \mathbf{1 \cdot 0 4}, \mathbf{I} \cdot 02, \mathbf{I} \cdot 00, \mathbf{0 . 9 7}, \mathbf{I} \cdot 00$, while the first gives $0.99, I \cdot 05, \mathbf{1} \cdot 06,0.99,0.98, \mathbf{0 . 9 4}$ respectively. Since the absorbability of the excited radia- 
tion is only about 3 per cent. greater than that of the exciting one, and is about inversely proportional to the sixth power of the atomic number, we get $v=2.23 \times 10^{8}(\mathrm{M}-\mathrm{I}) \mathrm{cm}$. $/ \mathrm{sec}$, while Bohr finds $(\mathrm{M}=\mathrm{N})$ :

$$
v=2 \cdot 18 \times 10^{8} \mathrm{~N} \mathrm{~cm} . / \mathrm{sec} \text {. }
$$

Now from this value of $v$, and $v=2.47 \times 10^{15}(\mathrm{M}-\mathrm{I})^{2}$, we can calculate $x$ from $x m v^{2}=2 h v$, which must be a constant, because both $v^{2}$ and $v$ depend on $(M-I)^{2}$. As $m v^{2} / 2$ is energy to be, at least in part, radiated away periodically, on the right side of the equation, not only the number of times energy is radiated away per second $(v)$, but also the total time of radiation $(t)$ and the mean energy radiated away per period (E) must occur, so that $x m v^{2}=2 t v \mathrm{E}$, and $t \mathrm{E}$ is a constant (which may mean only that the time during which radiation is emitted is inversely proportional, for a given frequency, to the quantity of energy that is radiated away during each period). Hence

$$
\begin{aligned}
& x=2 h v / m v^{2}=2.6 .62 \times 10^{-27} \cdot 2.47 \times 10^{15}(\mathrm{M}-1)^{2} / \\
& 0.88 \times 10^{-27} \cdot 2 \cdot 23^{2} \times 10^{16}(\mathrm{M}-1)^{2}=0.748, \text { or } 3 / 4,
\end{aligned}
$$

as assumed by Moseley.

From $m v^{2} / a=e^{2}(\mathrm{M}-\mathrm{I}) / a^{2}$ we can calculate mav $=e^{2} \cdot(\mathrm{M}-\mathrm{I}) / v=4 \cdot 78^{2} \times 10^{-20}(\mathrm{M}-\mathrm{I}) / 2 \cdot 23 \times 10^{8}(\mathrm{M}-\mathrm{I})$ $=\mathrm{I} \cdot 03 \times \mathrm{IO}^{-27}$, while $h / 2 \pi=6.62 \times \mathrm{IO}^{-27} / 2 \pi=\mathrm{I} .05 \times \mathrm{IO}^{-27}$, so that $m a v=h / 2 \pi$, as assumed by Bohr, and

$$
a=5 \cdot 12 \times 10^{-9}(\mathrm{M}-\mathrm{I})^{-1} \mathrm{~cm} \text {. }
$$

All this is in agreement with Bohr's theory.

As may be seen from a previous letter (NATURE, March 5, rgI4, p. 7), some properties of the elements depend not on the atomic but on the "periodic" number $\mathbf{P}=8 r \pm b(r$ is the number of horizontal rows preceding that of the element period of rare-earth elements not counted, and $p$ the maximum or positive valency). Now the sum of these electrons of valency may be easily seen to be for all regular (nonelementar) inorganic molecules an integer multiplum of eight. Hence the same holds for the sum of all $P$ electrons in these molecules (ions and rare-gasesatoms included). Affinity is then the tendency to build up systems of $8 n$ P-electrons, and, of course, if such a molecule breaks up into atoms with each similar systems of $8 n$ P-electrons, such ions must be formed as known from electrolysis. The great facility with which molecules like $\mathrm{H}_{2} \mathrm{O}, \mathrm{NH}_{3}, \mathrm{HCl}$, though neutral, are added to such systems, may be due to each of them, containing 8 P-electrons. According to Bohr, rings of electrons, whether belonging to one or to more atoms, may unite if the number of electrons in both is equal, so that rings of 2,4 , and ultimately 8 will be the most probable ( 16 only if the charge is very great).

Of course, the objections to the "Saturnian" atom hold for such systems also. Indeed, the structure of the periodic system as a whole, and the curious relation between the number of the non-periodic $(\mathrm{Q})$ elements, $\mathrm{H}, \mathrm{He}, \mathrm{Co}, \mathrm{Ni}, \mathrm{Rh}, \mathrm{Pd}$, and that of the horizontal rows in the periodic system : $2 / 1,2 / 2,2 / 3,4 / 3,4 / 4,4 / 5,6 / 5,6 / 6,6 / 7$, suggests systems of $n$ equal non-coplanar rings of 8 electrons surrounding one or more (even $n$ ), positive nuclei, with $n$ or $n \pm \mathrm{I}$ electrons in or near the axis, and additional rings of electrons of valency, rather than a Saturnian atom. But, generally speaking, Bohr's theory is not in disagreement with the atomic number hypothesis.

Gorsel (Holland), April ${ }_{5} 5$.

\section{Means of Collecting Eelworms.}

THE rhubarb, when cultivated as a field crop, is subject to a wasting disease, which, attacking the rootstock and causing it to decay, occasions considerable loss to the grower. The diseased tissue, when NO. 2323 , VOL. 93] examined, is frequently found to be infested with the stem eelworm, Tylenchus devastatrix, Kuhn, and, in districts where this disease is prevalent, a supply of Tylenchus material is at hand which, since the rhubarb is a perennial plant, is available not only in summer but during winter also.

When pieces of decaying rhubarb tissue are enclosed in a corked tube, any Tylenchus worms that are present migrate to the surface and, provided they have not been corked up too long, will, if placed in water, remain alive for weeks. Material can be obtained in quantity, and with very little delay, by placing pieces of thubarb in a strainer covered with fine gauze, and suspended in a vessel of water. The eelworms, forsaking their feeding-ground, wriggle through the muslin and accumulate in a writhing mass on the floor of the vessel. This water method, it may be added, is also useful in examining the eelworm fauna of soil samples, and provides a simple means of ascertaining $\mathrm{rough} 1 \mathrm{y}$ what forms are present.

When thus collected from rhubarb, the eelworms are usually mixed with sediment, but this defect can be remedied by placing the material, while still unsorted, in a porous vessel, such as a candlefilter, which, when placed in water, allows only living eelworms to pass through. A better method of cleansing the material, however, is obtained by taking advantage of the habit that eelworms have of climbing up capillary films when these are present. For this purpose, silk threads are employed, to each of which is suspended a blob of cotton-wool, the cotton-wool serving as a receptacle for holding the crude material obtained from the rhubarb. The upper ends of the threads are attached to a glass ring which is supported upon the sloping sides of a funnel-shaped vessel containing water-this shape being chosen in order that the blobs may hang clear.

As the threads become saturated, the eelworms, leaving all impurities behind in the cotton-wool, ascend amongst the silken strands, and, passing over the brim into the water, congregate on the floor of the vessel-a feat on their part which, besides providing the student with clean material, raises the question whether, in respect of their acrobatic accomplishments, eelworms vary to any appreciable extent; and, if so, whether the rough method here described can be extended so as to provide a means of sorting out one species from another, when two or more species are present in the material employed.

M. V. Lebour.

The University, Leeds.

T. H. TAYLOR.

\section{THE PROHIBITION OF EXPERIMENTS ON DOGS.}

THE Dogs' Protection Bill for the second reading of which 122 members of Parliament were induced to vote the other day is one of those measures which are born of ignorance and fostered on misrepresentation. All our knowledge of the functions of the body is fundamentally based on experiments which have been made upon dogs. The action of the heart and its nerves; the 